\title{
LECTEUR LE VIERGE
}

Poème et Tabou

Jean-Christophe Cavallin

In Press | « Libres cahiers pour la psychanalyse »

$2012 / 1 \mathrm{~N}^{\circ} 25 \mid$ pages 7 à 26

ISSN $1625-7480$

ISBN 9782848352312

Article disponible en ligne à l'adresse :

https://www.cairn.info/revue-libres-cahiers-pour-la-psychanalyse-2012-1-page-7.htm

\section{Pour citer cet article :}

Jean-Christophe Cavallin, «Lecteur le vierge. Poème et Tabou », Libres cahiers pour la psychanalyse 2012/1 ( $\left.\mathrm{N}^{\circ} 25\right), \mathrm{p} .7-26$.

DOI 10.3917/lcpp.025.0007

Distribution électronique Cairn.info pour In Press.

(C) In Press. Tous droits réservés pour tous pays.

La reproduction ou représentation de cet article, notamment par photocopie, n'est autorisée que dans les limites des conditions générales d'utilisation du site ou, le cas échéant, des conditions générales de la licence souscrite par votre établissement. Toute autre reproduction ou représentation, en tout ou partie, sous quelque forme et de quelque manière que ce soit, est interdite sauf accord préalable et écrit de l'éditeur, en dehors des cas prévus par la législation en vigueur en France. Il est précisé que son stockage dans une base de données est également interdit. 


\title{
Lecteur le vierge
}

\author{
Poème et Tabou
}

\section{Jean-Christophe Cavallin}

«Le délavage à la pierre ponce n'est pas une idée nouvelle : la marque japonaise Edwin prétend l'avoir utilisé dès 1975, et Marithé et François Girbaud l'ont breveté en 1978 ; mais Diesel est la première marque à lancer à grande échelle le concept du "usedjean", littéralement jean "pré-usé", délavé à la pierre ponce ou aux enzymes, élimé par sablage et déchiré à coups de lame de rasoir. »

$\mathrm{M}$ ALLARMÉ DISTINGUE DEUX ÉTATS DE LA LANGUE : d'un côté, la langue d'usage, abusée et bonne à tout, dont la prolixité nourrit « l'universel reportage »; de l'autre, celle des poètes ou celle que chaque poète doit chaque fois se recréer - hermétique et « reculée de l'usage ordinaire » (Houdar de La Motte) - pour son propre jeu somptuaire. La première, communicative, s'épuise dans sa valeur d'échange : elle sert au commerce des idées et des énoncés pragmatiques. La seconde ne sert à rien qu'à l'observation de ses propres rites : «Oui, c'est pour moi, pour moi que je fleuris déserte ${ }^{1}$. " Vestale d'elle-même et de sa

1. Mallarmé (1887), Hérodiade. 
vacuité, sa consécration l'exclut de tout échange linguistique (elle ne transmet pas de message - échange d'un signe pour une idée - ni ne saurait être traduite - échange d'un signe pour un signe). La langue est double comme Vénus : d'un côté, la prostituée qui fait commerce d'ellemême $^{2}$ (Venus volgivaga); de l'autre, la vierge farouche qui s'isole et s'excommunie (Venus Urania).

Pour figurer ce double état ou clivage linguistique entre une langue ouvrable (opératoire et ouverte) et un idiome désœuvré (festif et forclos), Mallarmé choisit l'image d'une vieille monnaie trouvée aux arènes de Lutèce :

La pièce de monnaie, exhumée aux arènes, présente, face, une figure sereine et, pile, le chiffre brutal universel ${ }^{3}$.

Cette pièce de monnaie, du fait même qu'elle n'a plus cours, va se retrouver au musée : au lieu de se la passer de main en main en n'en vérifiant que « le chiffre », on expose sa face oisive et on interroge sans but son visage silencieux ${ }^{4}$. Cette pratique dex-ception ou d' « isolement » de la parole définit l'acte poétique selon Mallarmé : le poète exhume les mots du langage courant (monnaie courante ou currency de nos échanges linguistiques) et les soustrait au commerce en en paralysant le sens («le chiffre brutal universel » : ce que chaque mot signifie, la valeur qui est la sienne dans le système linguistique). Du moment qu'on ne comprend plus l'usage que l'on devrait faire de tel mot soudain incompréhensible,

2. C'est une image de rapport mercenaire que Mallarmé emploie pour parler de l'usage courant du langage - un échange rétribué et (paradoxalement) muet : «À chacun suffirait peut-être, pour échanger toute pensée humaine, de prendre ou de mettre dans la main d'autrui en silence une pièce de monnaie, l'emploi élémentaire du discours dessert l'universel reportage dont, la Littérature exceptée, participe tout, entre les genres d'écrits contemporains. » (Avant-dire au Traité du verbe de René Ghil, 1886.)

3. Mallarmé, La Cour.

4. Entre la « pièce de monnaie » mercenaire de la note 2 (cf. supra) - celle qu'on s'échangeait en silence dans le langage quotidien - et cette "pièce de monnaie exhumée au arènes », la différence est absolue. L'une est utilisée, l'autre est ritualisée. De l'une ne vaut que le « chiffre » (la brutale valeur d'échange); de l'autre ne vaut que la face oisive (la pure valeur de jouissance). 
il nous paraît l'entendre pour la première fois. Nos yeux l'écoutent et le répètent comme s'ils ne l'avaient jamais vu :

Le vers qui de plusieurs vocables refait un mot total, neuf, étranger à la langue et comme incantatoire, achève cet isolement de la parole [...] et vous cause cette surprise de n'avoir jamais ouï tel fragment ordinaire d'élocution.

L'acte poétique « refait» le langage. Le langage de tous les jours - cette « langue à nu, vulgaire, dardée sur les carrefours », cette langue galvaudée qui sert à tous et à tout $-\mathrm{s}$ 'y refait une virginité : il semble à celui qui l'écoute «n'avoir jamais ouï tel fragment ordinaire d'élocution ». De la boue du langage « or-dinaire », le vers refait (extrait) l' « or ». La parole était une putain, offerte à tous les coins de rue, le vers en refait une vierge, qu'on voit pour la première fois et qu'on n'ose pas toucher. Privé de son sens commun, le mot redevient «vierge absence » : la «vierge absence éparse en cette solitude» du superbe Nénuphar blanc ${ }^{5}$. Il redevient res nullius et donc inappropriable. La parole ainsi refaite n'offre plus aucune prise à notre compréhension : on ne peut avoir avec elle aucun rapport intellectuel. Sa virginité retrouvée paralyse l'acte de lecture (l'action de saisir) et condamne le lecteur à une attitude purement passive : il ne (com)prend plus, il ne fait qu'ouïr. On reconnaît aisément, dans la paralysie de l'intellect provoquée par la " poésie pure », la pathologie singulière évoquée par Freud dans Le Tabou de la virginité. Devant cette langue qu'il lui semble «n'avoir jamais ouï[e] », notre esprit éprouve l'angoisse paralysante de la première fois : il ne sait pas comment la prendre - ou s'y prendre - et la déflorer.

5. Le Nénuphar blanc (1817) compare les mots poétiques - ces mots de tous les jours dont leur immersion dans le vers répare la virginité - à un archipel de "nénuphars clos » et « enveloppant de leur creuse blancheur un rien ». Dans la poésie, la parole se caractérise donc par son hermétisme ( « clos») et par sa vacuité : au sens propre, elle renferme un vide et consiste donc en une « vierge absence », si l'on veut bien jouer sur la fausse étymologie de ce dernier mot et l'entendre comme privation ou ablation du sens (ab-sensus). Chaque mot du vers est « absence » en tant que le sens s'en est absenté ; et « vierge » en tant que cette absence est « envelopp[ée] » d'un hymen sonore que l'oreille entend d'autant mieux que l'esprit parvient d'autant moins à en percer l'énigme vide. 
Une source possible de l'image mallarméenne de « la pièce de monnaie, exhumée aux arènes » peut nous aider à comprendre la raison de ce tabou et de cette paralysie. Dans son Traité sur la Virginité, Grégoire de Nysse commente la parabole de la « drachme perdue ${ }^{6} »$, qu'il faut chercher dans sa propre maison en balayant les ordures qui jonchent le sol afin d'exhumer " l'image du roi, non point entièrement perdue, mais cachée sous l'ordure ». Cette « auguste image du roi, empreinte dès l'origine sur la drachme de nos cœurs » se retrouve dans la « figure sereine » qu'on exhume des arènes dans le fragment de Mallarmé, cette «figure sereine » que le poète doit exhumer des mots de la langue en balayant les significations bavardes et les intentions pragmatiques qui l'ensevelissent et l'occultent. Dans le Traité de Grégoire de Nysse, la virginité est une consécration qui nous permet de redevenir semblable au «premier homme dans sa première vie » en restaurant «en son état primitif l'image divine actuellement cachée par la souillure de la chair », c'està-dire en retrouvant « cette beauté déiforme de l'âme, faite à l'imitation du prototype divin, qui s'est obscurcie en nous comme un morceau de fer par la rouille de la malice ». La virginité correspond donc, non pas à un état de disponibilité absolue, mais tout au contraire à un état de consécration divine : si la vierge est res nullius (chose de personne), c'est parce qu'elle est res dei (chose de dieu). Elle est marquée d'un sceau divin qui l'interdit à l'usage et la soustrait au commerce normal des hommes. Et ainsi, pour Mallarmé, de la « poésie pure », laquelle est rendue au Verbe - à la puissance neutralisante du Langage - et que la restauration de cette emprise première soustrait à la prise du lecteur (à la lecture comme acte de compréhension et de consommation), condamnant celui-ci à une série de méprises.

Cette virginité non profanable parce que consacrée à la divinité du Verbe et marquée du sceau divin (« la figure sereine ») rappelle les développements de Freud sur « la fixation de la libido sur le père » et sur l'« amertume hostile » que ressent la femme contre son premier partenaire sexuel, c'est-à-dire contre celui qui tente de profaner la consécration primitive de la libido à l'imago paternelle. Le premier homme n'est donc jamais «tout au plus que le second» (Freud). Afin d'éviter les

6. Luc, 15. 
représailles liées à cette tentative d'usurpation et afin de neutraliser la violence du tabou, on met en place un acte propitiatoire prenant la forme d'un sacrifice : le jus primae noctis (droit de cuissage) du seigneur du Moyen Âge, le sacrifice de l'hymen au « lingam en bois » (Inde) ou au « phallus en pierre » (Rome) représentant le dieu sont autant de manières de reconnaître que la future mariée est toujours déjà déflorée par un frayage archaïque (fixation œdipienne de la libido féminine) dont ce rituel de défloration préventive est une traduction symbolique.

Un tel rituel de déconsacration des vierges, étape préliminaire à leur appropriation par un époux humain (disponibilité pour une relation sexuelle légitime et ce que Freud appelle « une vie commune durable et heureuse »), rappelle la cérémonie des prémices au cours de laquelle les premières gerbes de la moisson sont offertes en sacrifice : selon la logique rituelle du pars pro toto, le sacrifice d'une partie (la part du dieu) rend possible la profanation (ou retour à l'usage commun) de tout le reste de la moisson, dont les hommes acquièrent ainsi le droit de disposer pour leur consommation propre. Le sacrifice des prémices réserve aux dieux « la fleur » de la moisson, tandis que revient aux hommes les fruits d'une terre préventivement déflorée. La glose à laquelle donna lieu la première lettre de la Bible hébraïque permet de construire une analogie entre ce rituel archaïque et l'acte de la lecture ou interprétation des textes. Le première lettre de la Genèse est non pas l'aleph (première lettre de l'alphabet) mais le beth. C'est parce que Dieu a excis la première lettre de l'incipit du texte saint que ce texte appartient désormais aux hommes : la lettre beth, qui signifie aussi « la maison », indique que le Livre est désormais la maison des hommes. L'excision de la lettre aleph (cette « entame » est la pars dei) en a opéré la défloration symbolique : il revient désormais aux hommes d'habiter et d'interpréter ce texte déconsacré, rendu au monde profane et d'en retirer les fruits (intellectuels et moraux) qui pourront leur être utiles.

Dans l'expérience quotidienne de la lecture, on retrouve une telle technique de propitiation de la puissance intacte du Verbe. La tendance du lecteur moyen - c'est-à-dire de tout lecteur - est de se garder contre la virginité dirimante du texte. Freud écrit : 
Dans un nombre considérable de cas, la femme reste frigide dans son premier mariage et s'y sent malheureuse, tandis qu'après la rupture de celui-ci elle devient pour son second mari une épouse heureuse et tendre.

La réaction archaïque à la défloration, l'hostilité amère liée à la profanation d'une libido fixée sur la figure du père, «s'est en quelque sorte épuisée sur le premier objet» - c'est-à-dire sur le premier mari, qui se trouve n'être en fait «tout au plus que le second» ou, si l'on veut, le premier (et malencontreux) substitut d'une fixation originaire. Tout lecteur a connu cette sensation pénible de ne pas réussir - comme on le dit si bien - à entrer dans un texte. Quelque chose (quelqu'un ?) ne prend pas. À une approche frontale, les signes opposent leur froideur; le plaisir qu'on se promettait est obstinément repoussé par la frigidité du texte. Tout se passe comme si l'acte de défloration que la lecture cherche à accomplir déclenchait, de la part du texte, " une réaction archaïque d'hostilité $[\ldots]$ se manifestant par des phénomènes d'inhibition dans la vie amoureuse du couple » que le texte forme avec son lecteur. Contre cette herméticité et cette frigidité, il existe une parade - un rituel propitiatoire - que chacun de nous connaît bien : il s'agit de se reporter à la volonté de l'auteur. On rebrousse chemin et on lit la préface du livre, qu'on avait d'abord négligée. Ou on cherche à se rappeler ce que l'on sait de ce Nom couché (par écrit) en pole position sur la première de couverture. Bref, on invente ce fantasme d'une première relation (celle de l'auteur et de son texte) qui aurait su déflorer et déconsacrer le Verbe en le réduisant au verbiage d'un simple Discours d'auteur. Dans la Vita Nuova, Dante revient sur son œuvre lyrique et déclare son intention de l'« aprire per la prosa », c'est-à-dire de l' « ouvrir par la prose » d'un autocommentaire. C'est la fonction (explicitée) que le fantasme du lecteur assigne à l'Auteur du texte dont il suppose la présence et interpose le fantôme entre son acte de lecture et la sacralité intouchable du Verbe : l'auteur est l'ouvreur du texte. Sans sa présomption de présence, le texte demeure hermétique. Le vouloir-dire auctorial, comme supposition minimale de sens, représente le premier frayage permettant de s'aventurer dans la forêt vierge du Verbe. Il est « le premier mari », celui qui a rompu la glace de la frigidité du texte et chauffé la place au lecteur. C'est parce que ce sont de secondes noces que le mariage du lecteur et du texte est un 
mariage tendre et heureux : la voie est déjà frayée, le sens est autorisé, le message cryptique est ouvert.

Dans Omphalos (1857), le naturaliste Philip Henry Gosse soutenait que - de même qu'Adam avait été créé avec un nombril qui n'était que le trompe-l'œil d'une fausse gestation et d'un faux enfantement - la terre portait dès l'instant de sa création «les traces d'une existence antérieure », c'est-à-dire tout ce qu'il fallait de vieilles pierres et de fossiles. Il s'agissait pour lui de défendre la généalogie de Moïse - et les quelque six mille ans que le patriarche donnait à la création - contre les découvertes des géologues qui avaient exhumé des entrailles de la terre les preuves d'une multitude de déluges et d'une antiquité qui donnait le vertige. À cette multiplication des siècles géologiques, Gosse répondait que les gisements fossiles témoignaient, non pas de l'écoulement de siècles incalculables, mais d'une ruse esthétique de l'artifex divin qui avait modelé dans les entrailles de la terre les marques d'un passé fictif afin d'effacer le moment de son acte créateur. Les fossiles remontant en deçà des six mille ans autorisés par une interprétation littérale de la Bible étaient des fossiles "prochroniques », c'est-à-dire de purs simulacres dont on ne pouvait rien conclure. La terre avait été créée, non pas du tout dans sa fleur, mais vieille et maquillée de rides.

Une version esthétisante de cette thèse conservatrice se trouvait déjà dans le Génie du Christianisme de Chateaubriand, dans le livre consacré aux « objections contre le système de Moïse ». Le chapitre s'appelle - magnifiquement - Jeunesse et vieillesse de la terre. Contre Buffon et les savants qui « considèr[ent] chacun des six jours de Moïse comme un long écoulement de siècles », l'apologiste rétorque que « Dieu a dû créer et a sans doute créé le monde avec toutes les marques de vétusté et de complément que nous lui voyons aujourd'hui » :

Si le monde n'eut été à la fois jeune et vieux, le grand, le sérieux, le moral, disparaissaient de la nature, car ces sentiments tiennent par essence aux choses antiques. Chaque site eût perdu ses merveilles. Le rocher en ruine n'eût plus pendu sur l'abîme avec ses longues graminées [...] Sans cette vieillesse originaire, il n'y aurait eu ni pompe ni majesté dans l'ouvrage de l'Éternel; et, ce qui ne saurait être, la nature, dans son innocence, eût été 
moins belle qu'elle ne l'est aujourd'hui dans sa corruption. Une insipide enfance de plantes, d'animaux, d'éléments, eût couronné une terre sans poésie. Mais Dieu ne fut pas un si méchant dessinateur des bocages d'Éden que les incrédules le prétendent.

En bon peintre paysagiste, Dieu a parsemé son tableau de ce qu'il y fallait de ruines sur lesquelles on puisse méditer. L'argument est inattendu, mais la conclusion est limpide : la création divine n'exista jamais « dans sa fleur »; elle naquit déjà usée. Quand Adam en prit possession au matin du monde, elle était déjà « de seconde main » et montrant des traces d'usure. Sous l'argument créationniste se sent la propitiation d'une hantise archaïque : celle de pénétrer dans une terre vierge, celle d'être le premier à en ouvrir les chemins. Dieu n'aurait ainsi permis à l'homme de devenir «maître et possesseur » de la Nature qu'après l'avoir déflorée et en avoir pris les prémices. La terre qu'il lui a donnée serait une terre d'occasion dont la surface et les entrailles témoigneraient du frayage - pénétration et retrait - d'un premier propriétaire.

Le sens auctorial que l'on présuppose dans un texte ressemble beaucoup à ces rêveries sur la «vieillesse originaire » de la terre. De même que la création parle de son créateur, dont l'absence est partout sensible ( Coeli enarrant gloriam dei... », « Les cieux proclament la gloire de Dieu... »), de même un texte ne saurait parler que par la voix de son maître, c'est-à-dire de son auteur. Son message (son vouloir-dire) est comme le « lingam de bois » ou comme le «phallus de pierre » qui défloraient la jeune épouse (y prélevaient la «part du dieu ») avant de la donner à son premier mari. Il est une espèce de viol propitiatoire, disant au lecteur que le texte ne saurait lui appartenir qu'à la condition d'y reconnaître la précédence d'un autre et de suivre la direction que cet autre a frayée pour lui.

Qu'en serait-il d'un poème sur lequel son auteur n'aurait pas exercé son jus primae noctis - c'est-à-dire un texte dont aucun vouloir-dire n'aurait défloré le mystère et sur la virginité duquel personne n'aurait laissé le sillage de ses empreintes? Un texte, donc, sur la page vierge duquel le lecteur ne pourrait pas suivre - comme une ligne pointillée - la direction tracée et le chemin frayé par le passage de l'auteur? Mallarmé 
tenta ce forfait ou ce retrait du poète ; il le tenta à maintes reprises et en figura la formule dans un de ses plus beaux sonnets :

Le vierge, le vivace et le bel aujourd'hui

Va-t-il nous déchirer avec un coup d'aile ivre

Ce lac dur oublié que hante sous le givre

Le transparent glacier des vols qui n'ont pas fui !

Un cygne d'autrefois se souvient que c'est lui

Magnifique mais qui sans espoir se délivre

Pour n'avoir pas chanté la région où vivre

Quand du stérile hiver a resplendi l'ennui.

Tout son col secouera cette blanche agonie

Par l'espace infligée à l'oiseau qui le nie,

Mais non l'horreur du sol où le plumage est pris.

Fantôme qu'à ce lieu son pur éclat assigne,

Il s'immobilise au songe froid de mépris

Que vêt parmi l'exil inutile le Cygne.

Dans un paysage de neige, un cygne est pris dans les glaces «pour n'avoir pas chanté ». Son silence est la raison de sa blanche captivité. «L'œuvre pure, écrit Mallarmé, implique la disparition élocutoire du poète $»$. Le cygne, imago traditionnelle du poète inspiré, a décidé de disparaître élocutoirement : il se fige au centre du poème, immobile et silencieux, retenant le « chant du cygne » qui romprait l'enchantement et réveillerait l'hiver qui pèse sur le sonnet. Ce mutisme du poète provoque une maladie, une pathologie des signes : l'auteur ne se prononce plus, il ne dit plus cette parole qui romprait l'ensorcellement de la virginité du texte, en ferait fondre l'énigme et dissoudrait au sens propre toutes ces paroles gelées que son silence a durcies dans leur insoluble froideur. Le cygne est pris dans un lac que son refus de chanter a enseveli sous le givre. Ce lac est « dur » comme on le dit d'un poème difficile. Il est fermé par la glace comme un poème « hermétique » et l'auteur-cygne tente en vain d'en déchirer la surface. Contrairement à Dante qui décidait d'ouvrir (aprire) par la glose son œuvre poétique, lui ne peut ouvrir 
l'« eau froide par l'ennui dans son cadre gelée » qui n'est autre que le sonnet au centre duquel il est pris.

D'où la figure énigmatique de ce « vierge [...] aujourd'hui » dont parle l'incipit. C'est quasi le sonnet qui parle, puisque l'auteur ne parle plus; et c'est le sonnet, ce «lac dur» qui aspire à ce que quelqu'un - finalement - le déchire. L'auteur est « cygne d'autrefois », il faut un cygne d'aujourd'hui qui saurait délivrer le lac de son froid linceul de givre. Mais « linceul » est incorrect; c'est « hymen » qu'il faudrait dire. Le poème que son auteur a refusé de déflorer attend sous un hymen de givre. Ce voile virginal, la dernière strophe l'appelle un vêtement de mépris (« songe froid de mépris / Que vêt parmi l'exil. ») - qu'il faut traduire : de méprise. En l'absence de l'auteur et de son autorité, un texte ne peut être compris et toutes les lectures possibles sont autant de virtualités ou de possibles mé-prises. L'aujourd'hui serait l'acte - enfin - qui saurait actualiser - rendre actuelles et effectives - ces oiseuses virtualités. Or, ce sens actuel du poème est une promesse ou un défi que le texte adresse au lecteur qui ose s'y aventurer : «Le vierge, le vivace et le bel aujourd'hui » est l'aujourd'hui de la lecture et il est vierge dans la mesure où personne ne l'a précédé dans l'entreprise périlleuse qu'il s'apprête à accomplir : c'est une « œuvre pure » qu'il devra fouler. Il pénètre en éclaireur le sein de ses blanches ténèbres que le poète n'éclaire plus du « sens clair » de son vouloir-dire. Dans ce dessein, pour être exact, il a eu des prédécesseurs. Certains sont venus avant lui. Le poème évoque un « lac dur»

... que hante sous le givre

Le transparent glacier des vols qui n'ont pas fui.

L'eau gelée du lac est hantée : maints « vols » y sont pris en glace, captifs de la fixité de son sein forclos et frigide. Reprenons l'histoire au début : le cygne n'a pas chanté et l'hiver s'est abattu, comme un sort ou un maléfice, sur ce paysage endormi. D'autres vols sont venus ensuite et ont tenté de briser (de déchirer «d'un coup d'aile ivre ») l'hymen de cette « eau froide [...] dans son cadre gelée ». Ils n'ont pas su la déflorer - pas su dissoudre sa froideur ou résoudre son énigme - et ont été engloutis dans son hermétisme frigide en châtiment de l'orgueil de ce viol 
inaccompli. La fable est aussi transparente que ce «transparent glacier » : l' « eau froide » du sonnet est une espèce d'Hydre (hydros/hydra), une de ces vierges monstrueuses assises sur le charnier où pourrissent les prétendants qui ne surent pas les « ouvrir» ou en résoudre (solvere : fondre, dégeler) l'énigme. On pense aux chantantes Sirènes accroupies sur des écueils jonchés des ossements de marins naufragés. On pense à la Sphinge thébaine et au parvis de sa caverne où se décharnent et pourrissent les cadavres de tous ceux qui n'ont pas su décrypter ses paroles énigmatiques. On pense encore à Turandot, la vierge «cinta di gel » (entourée de gel comme d'une enceinte - c'est-à-dire non déflorée) et à la longue série des princes décapités pour n'avoir pas su résoudre ( $\mathrm{sco}$ gliere) ses énigmes ${ }^{7}$. Le « lac dur» est décrit comme le «transparent glacier des vols qui n'ont pas fui ». De même que le mot de « lac » (« ce lac dur ») est pris dans celui de « G[lac]IER » (« le transparent glacier »), les vols prisonniers des glaces sont le charnier de tous ceux qui ont tenté jusque-là - jusqu'à ce « vierge [...] aujourd'hui »-de déflorer le poème et ont succombé dans cette entreprise.

Dans la fable de ce poème, c'est quelque chose qui ressemble au « tabou de la virginité » que Mallarmé réactive. En s'absentant de son sonnet (suicide élocutoire) et en le refermant derrière lui, le poète (re)crée une sorte d'angoisse de la page blanche à l'usage du lecteur. Le poème est appréhendé sans pouvoir être compris. L'appréhension (de la lecture) gèle et frappe de paralysie la compréhension (du texte). Le lecteur craint de s'approcher de cette Hydre de blancheur dont le «pur éclat » l'éblouit et démunit ses facultés : dans le sein réfrigéré de la vierge monstrueuse, il reconnaît le charnier de tous ceux qui, avant lui, ont échoué à l'ouvrir. Le maléfice est entier. La vulve forclose de l'hydre - ce «transparent glacier» hanté de ses victimes - demeure frigide et durcie. Un troupeau de « vols » amputés et « le plumage » du cygne sont prisonniers de cette « eau froide » qu'ils ont tenté de déchirer. Leurs efforts se sont brisés sur le seuil clos de cette vulve qu'ils n'ont pas su

7. Le verbe italien scogliere signifie à la fois résoudre et faire fondre. La foule appelle le prince inconnu le scioglitore degli enigmi (celui qui résout/dissout les énigmes) et la réponse à la dernière est justement : « Turandot »-cette vierge vêtue de gel qu'il lui aura donc fallu et « résoudre » comme énigme et « dégeler » comme frigide (dans les deux cas : scogliere). 
pénétrer. Elle a refermé sur eux ses mâchoires dentées de glace et conservé dans son sein cette partie d'eux-mêmes dont elle les a châtrés. L'image est transparente à l'instar du glacier où les vols sont captifs. Ce dont cette vulve hermétique a privé ceux qui ont voulu s'aventurer dans son sein, c'est du pouvoir de s'élever ou de se lever (leurs « vols »), soit leur puissance virile ou leur phallus érigé (le « plumage » captif du cygne serait la plume de l'auteur, symbole phallique de sa puissance sur lequel se sont refermées les lèvres givrées du poème).

C'est donc sur l'aile du lecteur, comme ultime alternative, que le poème doit s'envoler vers la "région où vivre », image des beaux climats et promesse d'un dégel des signes qui pourrait en délivrer la signification captive. Le «coup d'aile » dont on attend qu'il déchire l'hymen de givre qui protège cette eau noire est l'« aujourd'hui » de la lecture : c'est le défi que le poème jette au lecteur qui s'aventure. Dans son combat avec le Verbe, le poète a laissé des plumes : en même temps qu'il s'absentait - élocutoirement parlant - de l'espace de son poème, il y a laissé une aile et l'a dressée rituellement à l'entrée du premier vers pour que le lecteur s'en saisisse et brise la glace des signes. On sait combien Mallarmé prêtait d'attention à la « lettre d'attaque du vers»-cette « lettre d'attaque » qui avait, pour lui, « la même importance que la rime »- et regrettait la suppression de la majuscule qui la distinguait. Il appelait cette consonne « la clef allitérative » du vers. À l'instar d'une clef d'interprétation en tête d'une partition, cette consonne (et elle seule) permettait d'interpréter (au sens musical et intellectuel) les signes notés du poème. Dire que la consonne d'attaque est une "clef allitérative » revient à dire qu'elle permet d'ouvrir le système phonétique qui structure le poème, c'est-à-dire de suivre l'allitération principale à même de rassembler le réseau de phonèmes (et des mots qu'il relie entre eux) dans lesquels est déposé la « signifiance » du texte. Cette alliteratio princeps se répand d'un mot à l'autre comme « une traînée de feux sur des pierreries », allumant par contiguïté et contagion phonétique différents lieux du poème et créant un réseau de lumière au sein de son obscurité. Lisons les deux premiers vers :

Le vierge, le vivace et le bel [L] $]$ aujourd'hui

Va-t-il nous déchirer avec un coup d'aile $[\underline{L}]$ ivre... 
Un coup d' " $\mathrm{L}$ » ouvre le poème et le frappe encore deux fois comme pour en secouer la frigide et "blanche agonie ». Le " coup d'aile ivre» du deuxième vers est bien - pour l'oreille - un coup d' « L ». C'est donc armé de cette lettre, triplement inaugurale en tant qu'elle est à la fois la première lettre du sonnet (« Le vierge »), l'initiale du mot Lecteur et la première lettre de l'alphabet romain ${ }^{8}$, que le lecteur peut espérer briser l'obscurité frigide du poème et rompre l'enchantement qui y tient prisonnier l'auteur et les autres sens hasardés. Dans son ouvrage sur Les Mots anglais, Mallarmé écrit d'ailleurs du L considéré comme radical consonantique de multiples verbes et substantifs (lag, lug, lounge, lay, lie, lull, lump, etc.) :

$\mathrm{L}$, ne pouvant s'unir à l'autre liquide $r$, ni en tant que consonne initiale se redoubler, frappe, au commencement des mots, toujours une voyelle; et apparaît donc là dans toute son intégrité. Cette lettre semblerait parfois impuissante à exprimer par elle-même autre chose qu'une appétition point suivie de résultat, la lenteur, la stagnation de ce qui traîne ou gît ou même dure ${ }^{9}$.

Dans cette rêverie cratylienne à laquelle se livre le poète-linguiste, on remarque deux éléments essentiels. Le premier est que le $\mathrm{L}$ en position initiale, «ne pouvant s'unir » ni à une autre consonne ni à lui-même, $\mathrm{y}$ apparait toujours « dans toute son intégrité »: il est donc vierge quand il « frappe » la voyelle qui le suit - « vierge » comme ce « coup d'aile » (sic) dont le « bel aujourd'hui » doit frapper l'hymen gelé du poème. Le second est que la signification diffuse que Mallarmé donne à cette consonne radicale est celui d'une vélléité (ou " appétition») « impuissante » à réveiller « la lenteur, la stagnation de ce qui [...] dure ». Le moins que l'on puisse dire est que cette ébauche impressionniste ressemble beaucoup à une paraphrase de notre poème : le lac dur stagnant sous le givre, la blancheur immobile du paysage, l'espérance

8. L'alphabet romain commençait à la lettre $\mathrm{L}:$ l'elementum [L+M+N(-tum)] est l'« abécédaire » latin. Les « éléments » d'une science en sont les premiers principes : le « B, A, $\mathrm{BA} »$ ou l'« A, B, C».

9. Mallarmé (1877), Les Mots anglais, Livre I, chap. 1. 
impuissante d'un coup d'aile qui le frapperait, le cygne (et aussi bien : le signe) illisible blanc sur blanc, à la fois inutile et inutilisé.

Donnons donc les tout premiers tours - ou les tout premiers coups (« d'aile ») - de cette lettre apéritive ou « clef allitérative » dont le lecteur doit se saisir comme Énée du rameau d'or qui savait ouvrir les Enfers. On la retrouve par trois fois dans trois mots monosyllabiques dont la triade organise l'espace de tout le sonnet : le VOL (« les vols qui n'ont pas fui »), le COL («Tout son col secouera ») et le SOL («mais non l'horreur du sol où le plumage est pris »). Le col ébroué du cygne représente la tension entre le sol et le vol, entre le haut et le bas, entre la profondeur captive et l'altitude impossible ${ }^{10}$. Cette triade ouvre à son tour sur une nouvelle traînée allitérative dont le frayage est la trace et la promesse du sens cherché. Le cygne est « cygne d'autrefois» («Un cygne d'autrefois se souvient que c'est lui ») : en latin, cygne se dit olus (olus, oleris, n.) et autrefois se dit olim. Le cygne, autrefois, était donc olus; et « Un cygne d'autrefois » peut se traduire : Olus olim... Cette allitération in absentia, suggérée par le jeu précieux d'une énigme étymologique, enrichit de nouveaux reflets la traînée phonétique : vol [olus, olim,], col, sol.

L'œuvre pure implique la disparition élocutoire du poète, qui cède l'initiative aux mots, par le heurt de leur inégalité mobilisés ; ils s'allument de reflets réciproques comme une virtuelle traînée de feux sur des pierreries, remplaçant la respiration perceptible de l'ancien souffle lyrique ou la direction personnelle enthousiaste de la phrase ${ }^{11}$.

10. Notons qu'à cette suite allitérative, qui ressemble beaucoup à la table des radicaux consonnantiques que Mallarmé donne dans Les Mots anglais, vient s'ajouter le mot de « lac », espèce d'inversion du « $\mathrm{col}$ » qui cherche à s'en délivrer. Le mot « lac » commence d'ailleurs, comme le sonnet lui-même, par cette consonne L qui vient frapper - «d'un coup d'aile »- la voyelle radicale comme pour la ranimer de la stagnation que le mot exprime. Notons encore que ce mot de «lac» est l'homonyme d'un mot anglais (lack) qui signifie « manque » ou « absence de » : ce « lac dur»-autrement dit : ce carré de vers hermétiques - est bien une « creuse blancheur » (Nénuphar blanc), une neige de mots vides au référent aboli : lack thereof ou «absence de ». Gertrude Stein, dans Sentences, et peut-être en souvenir du « lac dur» de Mallarmé, écrivait - plus expéditive : A lake is an article followed by a noun (« Un lac est un article suivi d'un nom »). Radicale littéralité où, vierge de tout réalisme, le langage seul est en jeu.

11. Mallarmé (1897), Divagations, « Crise de vers ». 
Au lieu donc que le poète nous précède dans son poème et en foule la neige vierge afin d'y imprimer la « direction » à suivre, il s'y supprime comme éclaireur, en abolit le sens clair et « cède l'initiative aux mots » : la séquence «vol, [olus, olim,] col, sol», composée des rapports distants d'inégalités phonétiques mutuellement mobilisées, représente cette « virtuelle traînée de feux sur des pierreries » dont Mallarmé suggère qu'elle contient le sens du poème. Dans ce jeu de reflets, « les mots, d'eux-mêmes, s'exaltent à mainte facette [...], prompts tous, avant extinction, à une réciprocité de feux ${ }^{12}$ ». C'est alors que le véritable acte de lecture commence et que chaque lecteur doit postuler l'intelligibilité de ce circuit phonétique (ou dispositif de signes) et en gloser les rapports d'égalité et d'inégalité selon le principe que « toute similarité apparente dans le son doit être évaluée en termes de similarité et/ou de dissimilarité dans le sens ${ }^{13}$ - ce que Mallarmé appelle « la retrempe alternée en le sens et la sonorité » comme opération principale de la lecture poétique.

Mais je n'irai pas plus loin que le frayage phonétique de cette première « traînée de feux ». Mon propos n'est pas d'expliquer Le vierge..., mais de montrer que le modus operandi propre au travail de Mallarmé et à son idée d' ' œuvre pure » ressemble beaucoup moins à une défloration préventive du texte (à l'usage du lecteur) qu'à une opération inverse de chirurgie reconstructive : le poète, en s'en retirant, répare l'hymen de la Langue. Il refuse de lui imposer la violence nécessaire à son éducation, c'est-à-dire à sa sortie de l'enfance sémiotique et à son accession à l'ordre symbolique où les signes ne sont plus des choses, mais châtrés d'elles, les signifient. La poésie de Mallarmé est ce qui ressemble le plus, dans le champ du symbolique (c'est-à-dire d'un langage qui veut dire quelque chose), à ce que serait la Langue non encore pliée à l'ordre (symbolique) du père. Cette langue, non pas vierge ${ }^{14}$, mais à l'hymen restauré, fait retour indéfiniment vers la virginité protosymbolique des signes. Elle pointe indéfiniment vers cette Chair de la langue, hermétique

12. Mallarmé (1896), Le Mystère dans les Lettres.

13. Jakobson (1963), Essais de linguistique générale. Le linguiste parle de «pertinence du nexus son/sens ».

14. En tant qu'elle emploie des mots usés et déflorés par l'usage, c'est-à-dire des mots qui veulent dire quelque chose - ce « sens commun » témoignant de leur appartenance à l'ordre du symbolique. 
et inentamée, dont le Verbe créateur, dont on prétend qu'il l'a produite, n'est qu'un produit dérivé. Sa réticence suggère que c'est la Chair qui se fait Verbe ou refuse de se faire Verbe ${ }^{15}$. Au rebours de toute entreprise qui tenterait de la lier dans une lecture sémantiquement cohérente assurant la « vie commune durable » (et clairement monogamique) de tous les signes du texte avec chacun un sens unique, elle se rétracte sans fin dans son obscurité farouche, entre une hostilité obstinément frigide et la monstrueuse polysémie de «vols » sans fin avortés.

L'angoisse que le lecteur ressent à l'idée de pénétrer dans cette langue réfractaire dont nulle pensée d'auteur n'a châtré préventivement la violence sémiotique ressemble beaucoup à la hantise liée à l'infraction du «tabou de la virginité ». L'eau durcie du texte est une Hydre, une vierge monstrueuse refermée sur le charnier de ses prétendants vaincus. Freud fournit de ce tabou une lecture œdipienne qu'il déduit d'une observation clinique : « la réaction paradoxale de la femme à la défloration ». La hantise masculine se trouve donc justifiée par une « réaction paradoxale » (et potentiellement hostile) de la femme au premier rapport amoureux - réaction dont Freud retrace l'origine, en deçà $\mathrm{du}$ choix objectal œdipien, dans la configuration du « narcissisme originaire ${ }^{16} »$. Dans le contexte qui est le mien - celui de la lecture des textes -, à l'assise œdipienne se substitue de la même façon une assise narcissique. L'angoisse de « la première fois » cède le pas à celle d'être sans prédécesseur : angoissante relation d'inconnu qu'une psyché sans modèle entretient avec elle-même. Le vrai tabou (narcissique) serait celui qui pèse sur une identité que n'autorise ou ne légitime aucun processus d'identification - un moi qui ne serait pas un moi de seconde main, une identité que ne garantirait pas l'assimilation mimétique à une identité d'emprunt. C'est l'angoissante condition à laquelle Mallarmé condamne son lecteur - lequel ne peut plus prétendre identifier sa lecture à quelque « pensée d'auteur» (mens auctoris) qui la légitimerait, en

15. En favorisant le côté physique ou la physis des signes, Mallarmé contredit Et verbum caro factum est, clef de voûte de l'ordre symbolique. Dans sa pratique de la Langue comme physis ou natura naturans, c'est bien la Chair qui se fait (ou refuse de se faire) Verbe.

16. «Mais la phase virile de la femme, celle où elle envie le garçon pour son pénis, est plus ancienne dans l'histoire de son évolution et est plus proche du narcissisme originaire que l'amour objectal [i.e. : la fixation de la libido sur le père comme premier objet]. » 
fonderait la performance et en baliserait l'exercice de symbolisation (assigner une signification à sens unique à chaque signe du poème, c'està-dire : l'identifier).

Chateaubriand, qui croyait à la « vieillesse originaire » de la création, croyait de façon analogue à la vieillesse originaire du processus identitaire. Voici ce qu'il écrit, toujours dans le Génie du Christianisme, à propos du baptême :

Regardez ce jeune enfant sur les fontaines sacrées. Une famille pleine de joie l'environne ; elle renonce pour lui au péché ; elle lui donne le nom de son aïeul, qui devient immortel dans cette renaissance perpétuée par l'amour de race en race. [...] Des pleurs d'attendrissement et de religion coulent de tous les yeux; le nouveau nom de l'enfant, l'antique nom de son ancêtre, est répété de bouche en bouche ; et chacun, mêlant les souvenirs du passé aux joies présentes, croit reconnaître le vieillard dans le nouveau-né qui fait revivre sa mémoire.

La naissance morale de l'enfant (le baptême) correspond donc à la renaissance de son aïeul. Le don du prénom s'accompagne d'une transfiguration ou plus exactement d'une figuration, puisque l'enfant - avant ce don - ne jouissait d'aucun visage et ne ressemblait à rien. Il ne prend figure humaine qu'à travers un acte de reproduction. Comme c'étaient les «marques de vétusté » qui faisaient toute la moralité de la terre au jour de sa création, ce sont des marques de vétusté (la transparence de l'aïeul) qui opèrent la naissance morale de l'enfant dont l'âme est identifiée par le sacrement du baptême. On ne pourrait être soi-même qu'en passant derrière quelqu'un et en s'identifiant à lui. En nous résisterait l'inné - le besoin de reproduire. L'homme, paradoxalement, cet animal aventureux, ne saurait que passer après, suivre quelque guide, modèle ou précepte de conduite qui, figeant sa vie dans un moi d'emprunt, le délivreraient de son devenir.

Dans le rite bénédictin de la prise de vœu, l'abbé demande : «Le veux-tu? » Et le novice répond : « C'est Dieu qui le veut d'abord, et moi ensuite. » Ce beau deinde ego ne définit pas seulement le renoncement à la volonté personnelle inhérent à la vie monastique; il exprime un des 
désirs les plus chers de la psyché : ne pas passer en premier, ne pas inventer sa vie. Cela signifie avant tout que le moi ne peut rien vouloir s'il ne s'imagine un Autre - ou lui sous l'espèce d'un Autre - qui veut cette volonté et duquel il la copie. C'est la source de toute morale : «agis comme si la maxime de ton action devait être érigée en loi universelle »-, toute réglementation revenant par essence à une généralisation de l'agir et donc à une prohibition de faire quoi que ce soit que l'on serait seul à faire et dont on pourrait dire : " C'est moi (seul) qui le fais ${ }^{17}$. » Un exemple, parmi les moindres : cette hantise de l'acte vierge pourrait être la raison de la mode du " pré-usé », ces habits dont la confection comporte une étape d'usure technologique, à la pierre ponce et aux enzymes. L'industrie textile court-circuite le phénomène de la " fripe » et vend des articles de mode «de seconde main » ab origine. Tout se passe comme si ce vêtement que vous portez pour la première fois avait déjà été porté (industrie du "pré-porté » plutôt que du prêt-à-porter) : vous y trouvez des «marques de vétusté » qui portent le faux témoignage d'une espèce de passé fictif. En enfilant vos habits, vous entrez dans la peau d'un autre ou - à tous les sens - dans ses habit(ude)s. L'habit est bien un habitus : un style de vie prêt-à-vivre et comme déjà vécu. De même qu'Adam aurait été saisi d'angoisse à l'idée de porter la main sur une création intacte que Dieu n'aurait pas déflorée, de même de ces habits dans lesquels on ne se sent bien - traduire : que l'on ne sent siens - qu'à la condition d'y trouver les empreintes illusoires (et donc les preuves du passage) d'un premier propriétaire. L'industrie textile joue le jeu et s'ingénie à me faire croire que ces vêtements que je porte sortent, non pas de l'usine, mais d'une absente penderie. Deinde ego : [quelqu'un d'abord, et]... moi ensuite.

Le moi lui étant " haïssable » - c'est-à-dire source d'angoisses et constant creuset de conflits -, la morale classique avait mis en place une règle de comportement qui soignait la même hantise que les fausses fripes de nos devantures. On la retrouve souvent sous la plume des

17. Le principe universalisant du semper (un comportement valable toujours et pour tous) s'oppose diamétralement au principe singularisant du semel (une fois seulement), selon lequel tout acte est vierge, c'est-à-dire non répétant (sans précédent) et non répétable (sans suite). 
auteurs, et jusque dans la correspondance de Descartes à Christine de Suède, mais c'est dans Le Commerce du monde du chevalier de Méré qu'elle est formulée avec le plus d'élégance :

Je suis persuadé qu'en beaucoup d'occasions il n'est pas inutile de regarder ce qu'on fait comme une Comédie, et de s'imaginer qu'on joue un personnage de théâtre. Cette pensée empêche d'avoir rien trop à cœur, et donne ensuite une liberté de langage et d'action, qu'on n'a point quand on est troublé de crainte et d'inquiétude. Ce qui m'en plaît encore davantage, c'est qu'on ne s'abaisse que bien peu dans la disgrâce, et qu'on ne s'élève pas trop dans la prospérité. Du reste, je ne vois presque point de si malheureux rôle, qu'on ne lui puisse donner quelque sorte d'agrément lorsqu'on fait tout ce qui se peut pour le bien jouer.

Bien avant le Paradoxe du comédien de Diderot, Méré pose l'équation paradoxale selon laquelle on joue d'autant mieux sa vie qu'on ne la vit pas comme sienne, mais comme un rôle emprunté et qu'on est d'autant plus soi-même qu'on se délivre de sa propre identité. Pour agir avec aisance - c'est-à-dire en propitiant toute angoisse identitaire -, il faut faire comme si nous ne faisions que nous acquitter d'un rôle que nous n'avons pas écrit : non pas auteur, donc, mais acteur. Le propre d'un rôle est de pouvoir se prêter à un nombre infini d'incarnations et le propre d'un comédien est de toujours répéter : je répète, donc je suis. En feignant d'être un comédien, l' « honnête homme » de Méré entre dans la (vieille) peau ou dans la (fausse) vieille peau d'une existence déflorée. Son moi est un rôle de composition qui ne peut se poser lui-même qu'en com-posant avec un autre (ou posant un autre avec/avant lui) qu'il imite du mieux qu'il peut. Il s'acquitte de lui-même comme d'un rôle bien appris et son seul critère d'excellence - la valeur qui le définit et engage son identité - est la faculté mimétique de pouvoir jouer tous les rôles et d'être n'importe qui sans aucune espèce de spécialité. Mais nous sommes tous cet homme de Méré et ne voulons pas d'une vie qui serait une terre vierge ou une jolie maison neuve avec un carré de jardin aux arbrisseaux sous plastique. Nous prenons possession de notre identité comme d'une vieille maison, qu'on se contente de rajeunir d'un léger coup de peinture, mais où l'ancien propriétaire ou les anciens 
propriétaires ont laissé partout leur empreinte et le parfum de leur vie. Notre existence est (h)antée : nous nous greffons sur (de) l'ancien.

Une question pour finir, que nous laisserons en suspens, à preuve que correspond à la hantise de commencer une hantise de conclure. Qu'Adam ait eu un nombril - sorte d'enfance en trompe-l'œil - quand Dieu le créa de l'argile, Philip Henry Gosse l'assure et l'affaire paraît entendue. Une incertitude demeure quant à la femme qu'on lui donna pour lui tenir compagnie : la reçut-il dans sa fleur ou préalablement « usée » comme la terre des origines ? Quelqu'un y avait-il prélevé les prémices? Si le nombril d'Adam est une affaire classée, l'hymen d'Ève demeure une question « ouverte »- ce qui n'est pas bon signe s'agissant d'un hymen.

Dans l'Ébauche d'un serpent, Valéry classe l'affaire. La voix sinueuse du poème se promet d'effacer en Ève la « ressemblance exécrée » et de profaner sans remède la «Paternité voilée » qui sommeille dans son sein :

Je vais, je viens, je glisse, plonge,
Je disparais dans un cour pur!
Fut-il jamais de sein si dur
Qu'on n'y puisse loger un songe !

La haine atavique que la Genèse institue entre les reptiles et les filles d'Ève ${ }^{18}$ trouverait une explication dans le fait que s'épuiserait sur le serpent déflorateur le ressentiment archaïque que la libido fixée sur l'image (divine) du père réserve au « premier mari » et à son acte de profanation.

Jean-Christophe Cavallin Professeur de littérature à l'Université d'Aix-Marseille.

18. «Je mettrai inimitié entre toi et la femme, entre ta postérité et sa postérité : celle-ci t'écrasera la tête, et tu lui blesseras le talon. » (Genèse, 3.15). 